\title{
Gsdma3 regulates hair follicle differentiation via Wnt5a- mediated non-canonical Wnt signaling pathway
}

\author{
Long He ${ }^{1}$, Mingxing Lei ${ }^{2,3}$, Yizhan Xing ${ }^{4}$, Yuhong $\mathrm{Li}^{4}$, Chunyan $\mathrm{Hu}^{4}$, Peixing Chen ${ }^{1}$, \\ Xiaohua Lian ${ }^{4}$, Tian Yang ${ }^{4}$, Wanqian Liu ${ }^{1}$ and Li Yang ${ }^{1}$ \\ 1"111" Project Laboratory of Biomechanics and Tissue Repair \& Key Laboratory of Biorheological Science and Technology of \\ Ministry of Education, College of Bioengineering, Chongqing University, Chongqing 400044, China \\ ${ }^{2}$ Integrative Stem Cell Center, China Medical University Hospital, China Medical University, Taichung 40402, Taiwan \\ ${ }^{3}$ Institute of New Drug Development, College of Biopharmaceutical and Food Sciences, China Medical University, Taichung \\ 40402, Taiwan \\ ${ }^{4}$ Department of Cell Biology, Third Military Medical University, Chongqing 400038, China \\ Correspondence to: Wanqian Liu, email: wqliu@cqu.edu.cn \\ Li Yang, email: yanglibme@cqu.edu.cn \\ Mingxing Lei, email: Imxgoodluck@163.com
}

Keywords: non-canonical Wnt signaling pathway; Gsdma3, hair follicle; epithelial-mesenchymal interaction; differentiation Received: July 18, $2017 \quad$ Accepted: September 21, 2017 Published: October 31, 2017

Copyright: $\mathrm{He}$ et al. This is an open-access article distributed under the terms of the Creative Commons Attribution License 3.0 ( CC BY 3.0), which permits unrestricted use, distribution, and reproduction in any medium, provided the original author and source are credited.

\section{ABSTRACT}

Hair follicle is a mini-organ that consists of complex but well-organized structures, which are differentiated from hair follicle progenitor or stem cells. How non-canonical Wnt signaling pathway is involved in regulating hair follicle differentiation remains elusive. Here we showed that Wnt5a regulates hair follicle differentiation through an epithelial-mesenchymal interaction mechanism in mice. We first observed that Wnt5a is expressed in the epithelial and dermal papilla cells during hair follicle development and growth. For the upstream of Wnt5a, RT-PCR and immunohistochemistry staining showed that Wnt5a expression is significantly decreased in the Gsdma3-mutant mice in vivo. Overexpression of Gsdma3 results in a significantly increased expression of Wnt5a in the cultured epidermal cells in vitro. We also checked the downstream factors of Wnt5a by adenovirus-mediated overexpression of Wnt5a to the dermal papilla cells isolated from the mouse whisker. We found that overexpression of Wnt5a suppresses canonical Wnt signaling pathway effectors such as $\beta$-catenin and Lef1. In addition, genes involved in maintaining cell quiescent state are also significantly decreased in their expression to the DP cells which were treated by Wnt5a. Our study indicates that Wnt5a mediates epithelia-expressed Gsdma3 to influence DP cell behaviors, which in turn regulate hair follicle epithelia differentiation in mice.

\section{INTRODUCTION}

Ectodermal organs consist of two important cellular partitions including epithelium and mesenchyme, which develop from different germ layers during early developmental stages but influence each other by direct cell-cell interactions or paracrine factors such as growth factors and extracellular matrix [1]. Epithelialmesenchymal interactions function virtually in every step of ectodermal organogenesis. Hair follicle positions as a consummate and highly tractable model to study epithelialmesenchymal interactions. It includes both epithelial tissues and a group of specified mesenchymal cells known as dermal papilla (DP) [2]. Epithelial-mesenchymal interactions play roles in nearly all development, growth and regeneration stages of hair follicle, to coordinate the formation of complex cellular components and structures of the follicle. Reciprocal signaling between the epidermal 
progenitor cells and the specialized dermal cells leads to the formation of hair placode and dermal condensate, which together grow downward to form a follicle in the dermis [2, 3]. After development, hair follicle undergoes cyclic growth consisting of anagen, catagen and telogen phases [4-6].

Several pathways have been identified to be essential for hair follicle development. Among them, Wnt signaling emerges as one of the earliest and mostimportant pathways [7]. Nineteen Wnt ligands have been discovered in human and mouse tissues. These Wnt ligands are classified to mediate canonical Wnt signaling pathway or non-canonical Wnt signaling pathway, both of which exert extensive physiological roles in governing cellular behaviors through an autocrine or paracrine mechanism during hair follicle development. For example, Wnt signaling pathway functions on specifying hair stem cell fate through a Wnt-SHH antagonism [8]. The canonical Wnt signaling pathway which utilizes $\beta$-catenin to relay the signal has been more studied in hair biology $[7,9$ 12]. Non-canonical Wnt signaling pathways are mainly categorized into three pathways, including the Wnt-cGMP/ $\mathrm{Ca} 2+$ pathway, Wnt/planar cell polarity (PCP) pathway, and the Wnt-receptor tyrosine kinase-like orphan receptor 2 pathway [13]. Little is known how non-canonical Wnt signaling is mediated via epithelial-mesenchymal interactions to commit hair follicle development.

$W n t 5 a$ plays widely roles in regulating cellular behaviors in many tissues. Combined with RorDishevelled, Wnt5a constitutes a core non-canonical Wnt pathway to regulate tissue morphogenesis during embryonic development [14]. Wnt5a is able to enhance mammary epithelial cell growth through receptor-like tyrosine kinase (RYK) [15]. It maintains hematopoietic stem cells in a quiescence state through suppressing Wnt3a-mediated canonical Wnt signaling pathway [16]. Mutation of $W n t 5 a$ results in increased angiogenesis through non-canonical Wnt signaling pathway [17]. In the hair follicle, Wnt5a is expressed in both epithelia and mesenchyme during hair development and cycling [18]. While Wnt5a-null mice display no apparent defect in embryonic hair follicle morphogenesis [19], overexpression of Wnt5a causes a delayed hair regeneration through inhibiting the canonical Wnt signaling pathway $[13,20,21]$. Recent study shows that adenovirus-mediated overexpression of Wnt5a in the cultured whisker results in a shortened hair shaft in length [22]. Mesenchymal Wnt5a mediates epithelial Notch/CSL signaling to control keratinocytes differentiation in the hair follicle [23]. These studies indicate that Wnt5a is largely associated with maintenance of stem cell fate, and inhibits hair follicle differentiation. Whereas the mechanism that how $W n t 5 a$ regulates cell differentiation in the hair follicle remains largely unknown.

GasderminA3 (Gsdma3) as a key member of gasdermin gene family is specifically expressed in the epithelial tissues, including mouse skin keratinocytes [24]. Gsdma3-mutant mice display disorders in hair cycling, with hair loss during postnatal life [25-27]. We recently uncovered that disorders during catagen-telogen transition in Gsdma3-mutant mice is attributed to increased canonical Wnt signaling in the epithelia of hair follicle [24]. The increased expression of $W n t 10 b$ promotes cell proliferation in the epithelial strand, resulting in a failure of telogen entry of hair follicles in Gsdma3-mutant mice. Gsdma3 also regulates differentiation of epithelial tissues in the skin, such as the hair follicles [27]. Mutation of Gsdma 3 causes a loss of interlocking structures, leading to the defects in hair shaft anchoring to the follicle. However, the molecular mechanism that how Gsdma3 regulates hair follicle differentiation remains unclear.

In the present study, we first examined the expression of Wnt5a and Gsdma3 in hair follicle in mice. To check the impact of loss of function of Gsdma3 on $W n t 5 a$ expression, we compared Wnt5a expression in the hair follicle of Gsdma3-mutant mice and wild type mice. We also investigated the effect of gain of function of Gsdma3 on Wnt5a expression in epidermal cells. By applying adenovirus-mediated overexpression of $W n t 5 a$ to the isolated dermal papilla cells, we evaluated the downstream targets of Wnt5a that mediate the noncanonical Wnt signaling in the DP cells. Our study demonstrates that epithelial Gsdma3 positively regulates expression of $W n t 5 a$, which influences the DP cell behaviors that further impact epithelial cell differentiation in an epithelial-mesenchymal interaction mechanism.

\section{RESULTS}

\section{Wnt5a expression during hair development}

Hair follicle develops multiple components at embryonic day 16.5 (E16.5), and acquires its complete structures at postnatal day 8 (P8) (Figure 1A-1B). At E16.5, immunofluorescence staining shows that Wnt5a is expressed in the mesenchymal components, particularly in the dermal condensation (DC) of the developing hair follicle (Figure 1C). When hair follicle enters full anagen at P8, immunohistochemistry staining shows that Wnt5a is expressed at a high level in the inner root sheath (IRS), and a lower level in the outer root sheath (ORS), dermal papilla (DP) and hair matrix (Figure 1D).

\section{Wnt5a is decreased in Gsdma3-mutant mice in anagen phase}

We recently found that Gsdma3 is also expressed in the hair follicle [24]. With a similar expression pattern to Wnt5a, Gsdma3 also shows an elevated expression in the IRS, and a reduced expression in ORS of the hair follicle (Figure 2A). Therefore, we speculated that there is an interaction between Gsdma3 and Wnt5a. To verify this, we 
harvested skin from WT mice and Gsdma3-mutant mice at the full anagen phase at P8. RT-PCR and its analysis show that the mRNA expression of Wnt5a is significantly decreased in the Gsdma3-mutant mice when compared to the WT mice (Figure 2B). Immunohistochemistry staining shows that Wnt5a is decreased in its expression to the IRS, ORS and hair matrix regions of the hair follicle in Gsdma3-mutant mice, compared to that of WT mice (Figure 2C). This suggests that the expression level of Wnt5a may be regulated by Gsdma3.

\section{Overexpression of Gsdma3 promotes Wnt5a expression in epidermal cells}

To examine if Gsdma3 regulates $W n t 5 a$ in epithelial cells, we constructed Gsdma3 overexpression plasmids and transfected them into the cultured JB6 cells. Green fluorescence shows successful transfection of Gsdma3expressing plasmid (Gsdma3+) and the control plasmid (Gsdma3-) into JB6 cells 24h after culture (Figure 3A). At this time point, RT-PCR and its analysis show that Wnt5a expression is significantly increased in Gsdma3overexpressed JB6 cells, when compared to the control group (Figure 3B-3C). These results further suggest that Gsdma3 may regulate and maintain $W n t 5 a$ expression levels within a certain range.

\section{Overexpression of Wnt5a suppresses Wnt signaling in DP cells}

Recent study shows that the formation of seven concentric differentiating epithelial layers of the hair follicle is the result of epithelial-DP interaction [28]. While Gsdma3 is expressed exclusively in epithelial
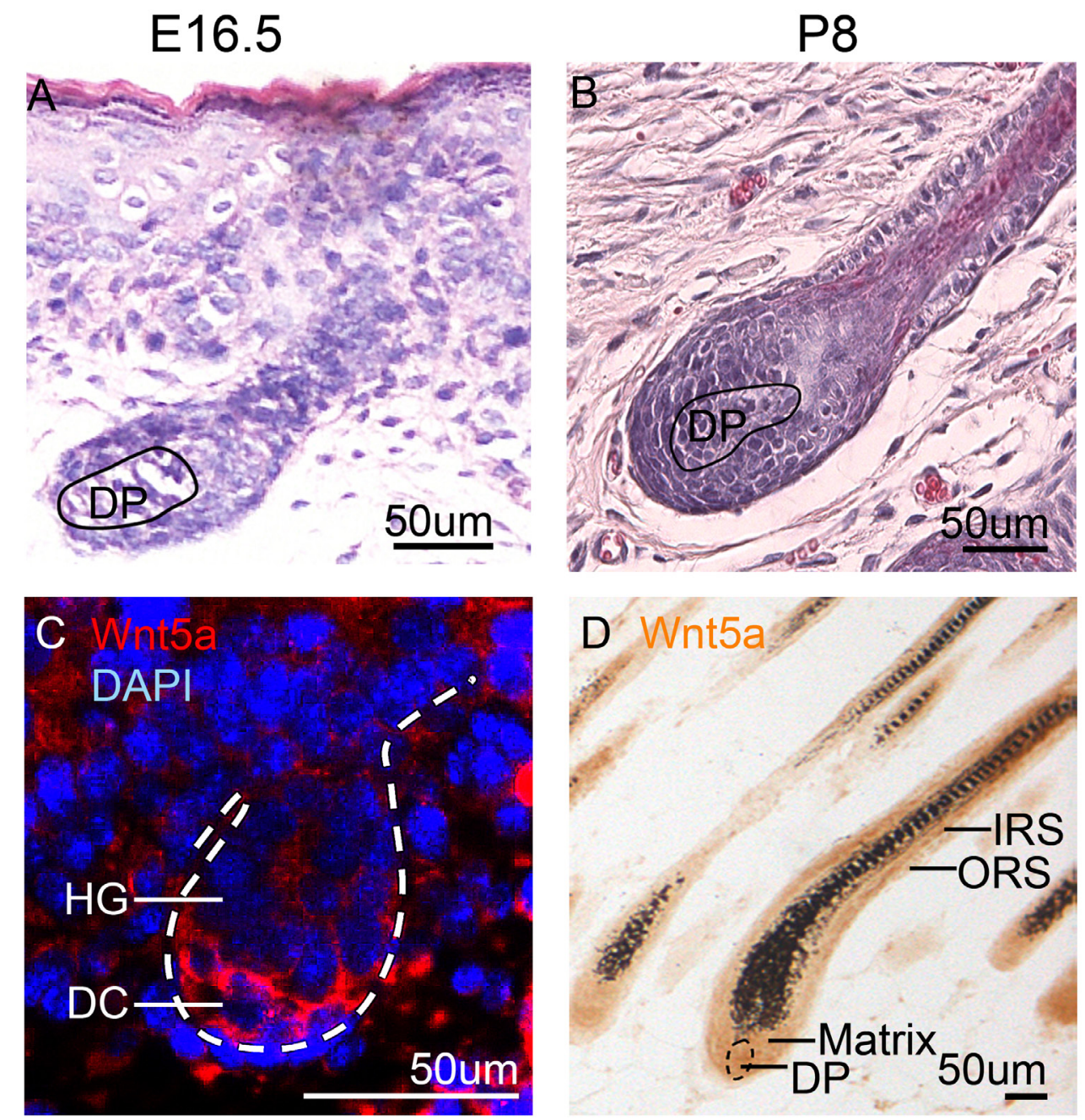

Figure 1: Wnt5a expression during hair development and growth. (A) H\&E staining shows a developing hair follicle at E16.5. (B) H\&E staining shows a developed hair follicle in full anagen at P8. (C) Immunofluorescent staining shows that Wnt5a is expressed in the mesenchymal cells of skin, particularly in the DP at E16.5. (D) Immunohistochemistry staining shows that Wnt5a is expressed in the IRS, ORS, hair matrix and DP cells. E16.5, embryonic day 16.5; P8, postnatal day 8; DC, dermal condensation; DP, dermal papilla; HG, hair germ; IRS, inner root sheath; ORS, outer root sheath. $\mathrm{N}=9$. 
cells [29]. To verify how epithelia-expressed Gsdma3 regulates $W n t 5 a$ expression to impact DP cells, we first isolated DP cells from the mouse whisker. DP-derived cells from the whisker show a different shape, compared to the epithelial-derived cells from the bulge of the hair follicle. When passaged for 1 time (P1), DP-derived cells display spindle shape with more filopodia (Figure 4A-4B), whereas epithelial-derived cells show an oval shape with more lamellipodia (Figure 4C-4D). 80\% the P1 cells show aSMA-positive and alkaline phosphatase (AP)-positive, indicating at least several cells have maintained the DP characteristics during culture (Figure 4E-4H).

We then overexpressed Wnt5a by applying Wnt5aexpressing adenovirus (AdWnt5a) into the P1 DP cells. Green fluorescence shows successful infection of AdWnt5a and the control adenovirus (AdGFP) into DP cells $24 \mathrm{~h}$ after culture (Figure 5A). However, real-time
PCR results show that the mRNA expression of Wnt pathway receptors including Fzds 1, 2, 3, 4, 6, 7, 8, 9, and 10, Lrps 5 and 6 are not significantly changed in the AdWnt5a-treated group, compared to the AdGFP-treated group $24 \mathrm{~h}$ and $48 \mathrm{~h}$ after culture (Figure 5B-5C). This indicates that Wnt5a cannot increase the expression of frizzled receptors in cultured DP cells. Intriguingly, RTPCR and its analysis show that the expression of key genes in canonical Wnt signaling pathway such as $\beta$-catenin and Lef1 is significantly decreased in DP cells after AdWnt5a treatment, when compared to the control group (Figure 5D-5E).

P53 gene has been reported to play an important role in cell cycle regulation [30]. P21 is a downstream target which acts as a regulator of cell cycle progression at G1 and S phase [31]. Overexpression of Wnt5a significantly decreased P53 expression but didn't influence P21
A

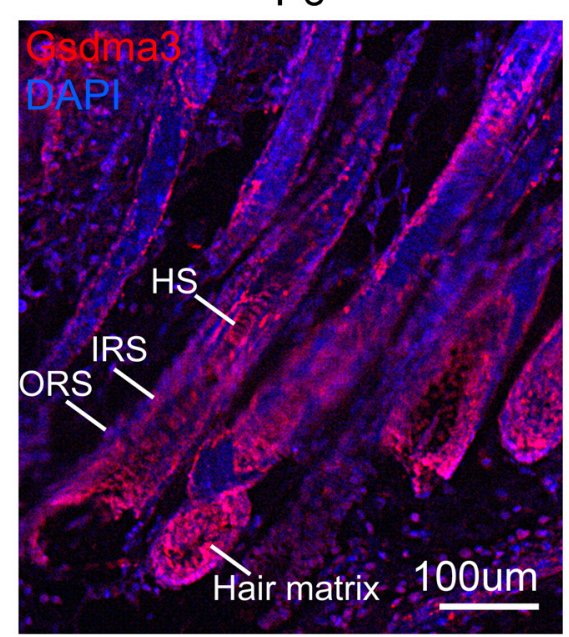

C

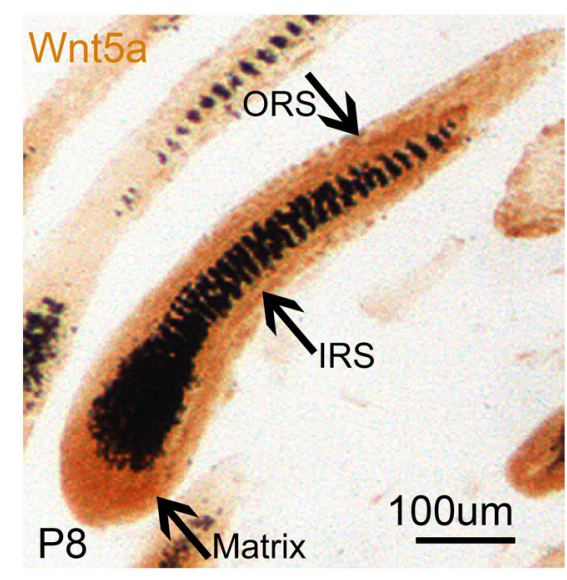

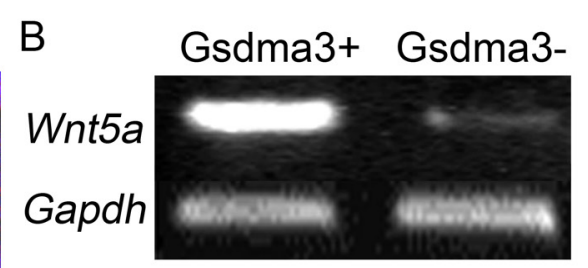

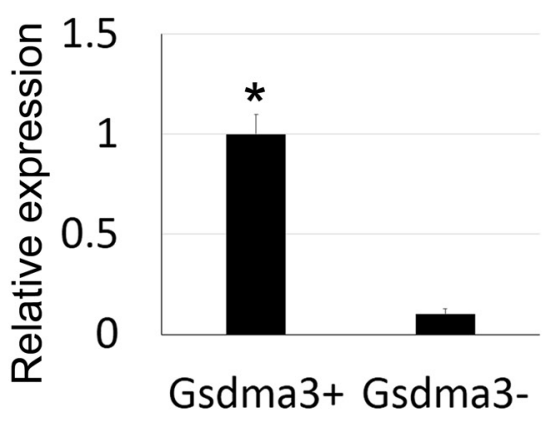

Gsdma3-

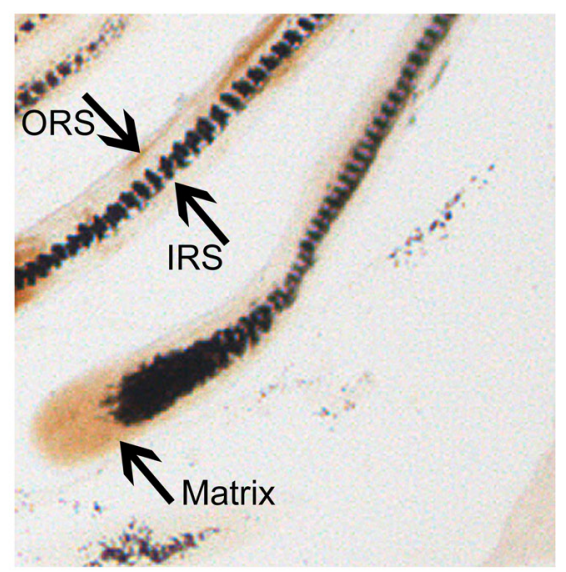

Figure 2: Decreased Wnt5a expression in Gsdma3-mutant mice. (A) Immunofluorescent staining shows that Gsdma3 is expressed in the IRS, ORS, hair shaft and hair matrix of the hair follicle in mice. (B) RT-PCR and analysis reveal that mRNA of Wnt5a expression is significantly decreased in Gsdma3-mutant mice skin when compared to the wild type mice. (C) Immunohistochemistry staining shows that Wnt5a expression is decreased in Gsdma3-mutant mice at ORS, IRS and hair matrix region at mid-anagen phase. P8, postnatal day 8; IRS, inner root sheath; ORS, outer root sheath. $\mathrm{N}=9$. 
expression (Figure 5D-5E). In addition, overexpression of $W n t 5 a$ results in significantly decreased expression of cell cycle genes such as $C y c l i n B 1$ and $C D K 1$ expression in DP cells, when compared to the AdGFP control group (Figure 5D-5E).

\section{DISCUSSION}

Increasing evidence shows that non-canonical Wnt signaling pathway is critical for hair follicle development, growth and regeneration. As a key non-canonical Wnt pathway ligand, Wnt $5 a$ functions as downstream of Sonic Hedgehog pathway to regulate hair follicle morphogenesis [18]. Wnt5a inhibits hair shaft growth during organ culture [22], and prevents hair follicles entering from telogen to anagen phase during hair regeneration through inhibiting canonical Wnt signaling pathway $[13,20,21]$. We previously proposed that an activator-inhibitor mechanism that regulates hair regeneration during aging [4]. In this study, we show that $W n t 5 a$ may act as a downstream of Gsdma3 to control hair follicle differentiation through an epithelial-mesenchymal interaction mechanism (Figure 6). Specifically, we demonstrate that Gsdma3 promotes Wnt5a-mediated non-canonical Wnt signaling pathway to orchestrate hair follicle differentiation.

Our group has uncovered that Gsdma3 is expressed in the IRS and hair shaft of the hair follicle [24], and is required for hair follicle differentiation [27]. Wnt5a which has the similar expression pattern with Gsdma3, is down-regulated in the Gsdma3-mutant mice, suggesting that $G s d m a 3$ may positively regulate $W n t 5 a$ expression in the differentiated layers of the hair follicle. There are at least two possible mechanisms that Gsdma3 regulates $W n t 5 a$ to influence hair differentiation. First, the intrinsic mechanism. Our data show that both Gsdma3 and Wnt5a are expressed in the epithelial cells including in the hair shaft and IRS of the hair follicle, and overexpression of Gsdma3 causes Wnt5a up-regulation in skin epidermal cells. It is possible that up-regulated $W n t 5 a$ leads to the differentiation of the local epithelial cells in the IRS and hair matrix. Given this is true, local epithelia-derived $W n t 5 a$ should directly promote hair follicle differentiation.
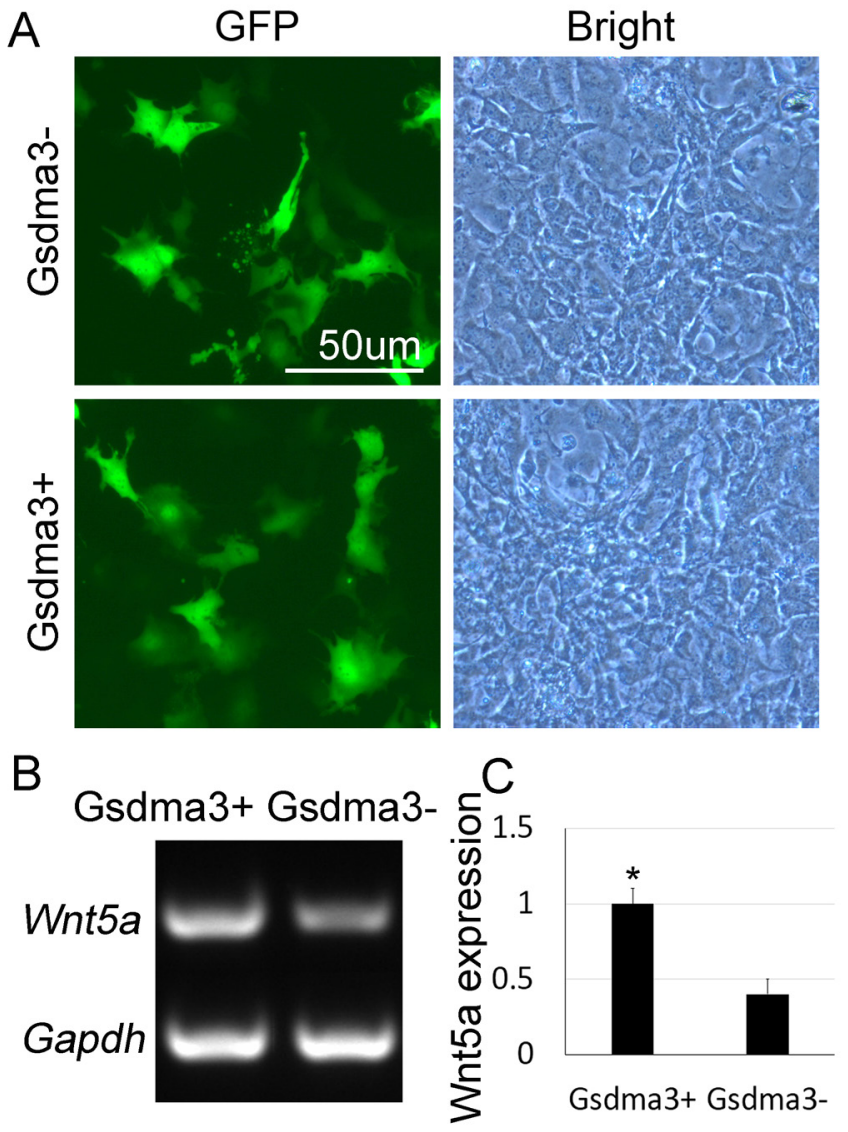

Figure 3: Overexpression of Gsdma3 up-regulates Wnt5a mRNA expression in epidermal cells. (A) Green fluorescence indicates successful transfection of Gsdma3 overexpression plasmid and control plasmid. (B) RT-PCR shows mRNA expression Wnt5a after Gsdma3 overexpression in epidermal cells. (C) Statistical analysis reveals that Wnt5a mRNA expression is significantly increased after in Gsdma3 overexpression group, compared to the control group. Gsdma3+, Gsdma3 overexpression group; Gsdma3-, control group. ${ }^{*} \mathrm{P}<0.5 . \mathrm{N}=9$. 
However, overexpression of Wnt5a in the hair follicle epithelia results in a shortened hair shaft [22] and a delayed hair regeneration [13], indicating epithelial $W n t 5 a$ may prevent rather than promoting hair follicle differentiation. Thus, we tend to speculate that the second mechanism that $W n t 5 a$ regulates hair follicle differentiation through the epithelial-mesenchymal interaction.

Epithelial-mesenchymal interactions are essential for hair follicle development, regeneration and function. Complex structures of the hair follicle form as a result of sequential, coordinated and reciprocal interaction between epithelium and mesenchyme. Recent studies showed that there are four molecularly distinct cell populations located in the dermal papilla [28]. They constitute micro-niches with the juxtaposed epithelial transient amplifying cells to govern the differentiation of keratinocytes into the seven concentric differentiating layers of the hair follicle. Transcriptome analysis revealed that $W n t 5 a$ is not only expressed in the DP, but also a signature of the cortex of the hair shaft. Indeed, Wnt $10 \mathrm{~b}$ as one of the ligands mediating canonical Wnt pathway, is expressed in the transient amplifying cells that give rise to the IRS. Whereas nuclear Lef1 as the proxy of Wnt signaling, is expressed in both IRS lineages and midDP. The interaction between epithelial and DP regulates IRS differentiation during hair growth. While Wnt5a is expressed both in epithelial and DP, Gsdma3 is specifically expressed in the epithelial tissues [29]. Thus, similar to $W n t 10 b$, it is tempting to speculate that Gsdma3 promotes $W n t 5 a$ expression in the epithelia, then epithelial- $W n t 5 a$ influences DP cells which in turn regulate differentiation of epithelial transient amplifying cell through epithelialDP interaction.

Wnt5a functions as a downstream mediator of Notch/CSL signaling in the DP to regulate the hair follicle differentiation [23]. How does Wnt5a influence DP cells
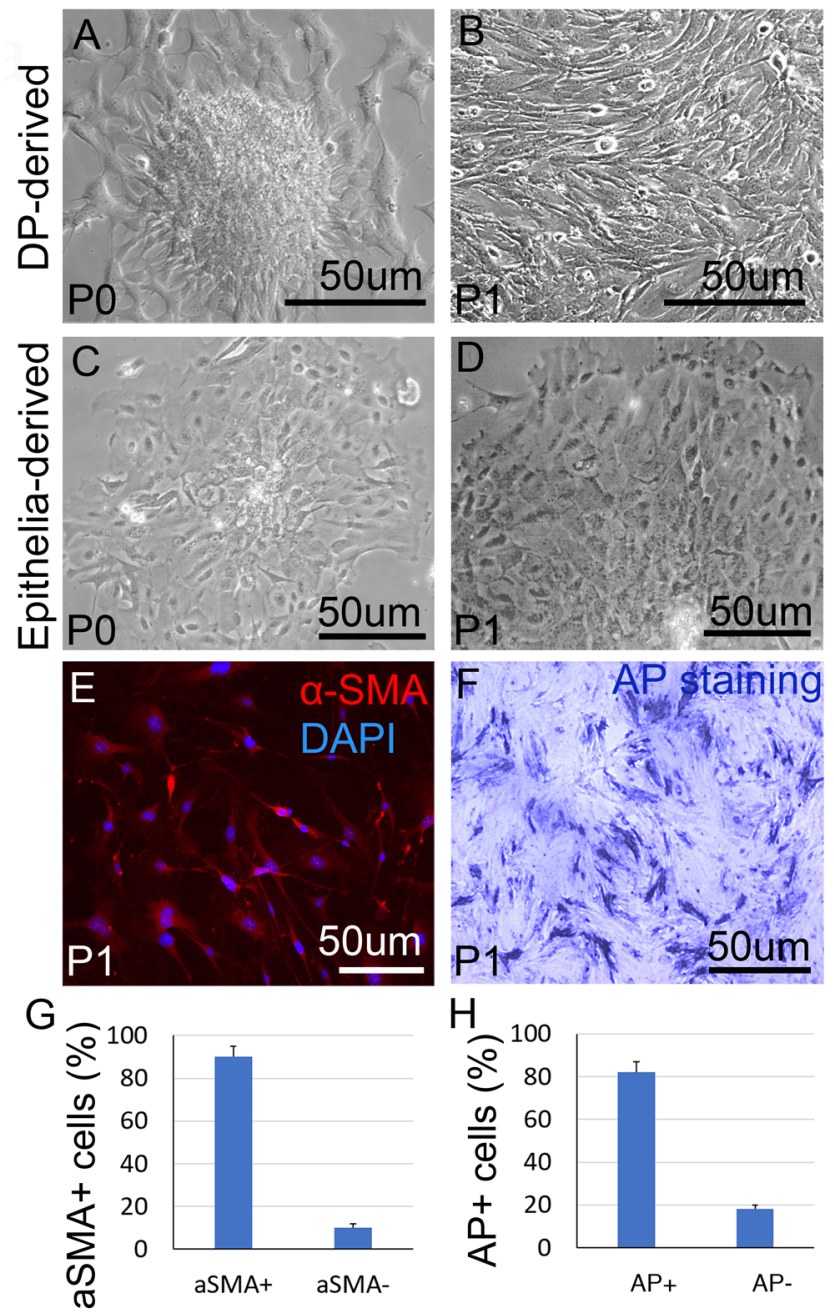

Figure 4: DP cell culture. (A) A DP from mouse whisker is expanded during culture. (B) DP cells grow in a spindle shape after 1 passage culture. (C) Primary epithelial cell culture from mouse hair follicle. (D) Epithelial cells maintain their morphology after 1 passage culture. (E) Immunostaining shows positive a-SMA expression in cultured DP cells. (F) DP cells show positive alkaline phosphatase staining after 1 passage culture. (G) Statistical analysis reveals that more than $80 \%$ P1 cells are aSMA positive; (H) Statistical analysis reveals that about $80 \% \mathrm{P} 1$ cells are alkaline phosphatase (AP) positive; $\mathrm{P} 1$, subculture for 1 passage. $\mathrm{N}=9$. 
A
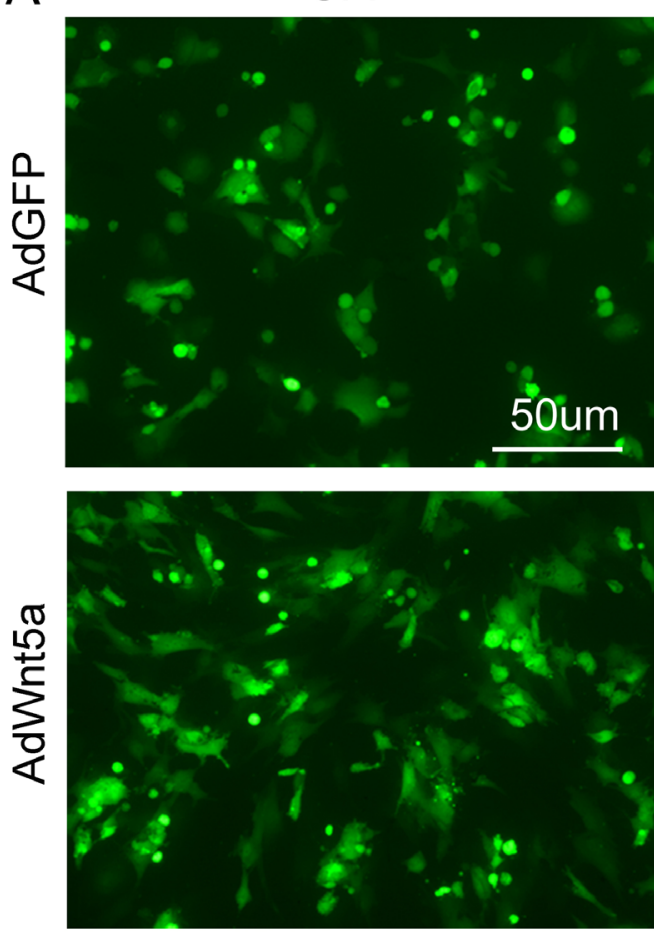

B

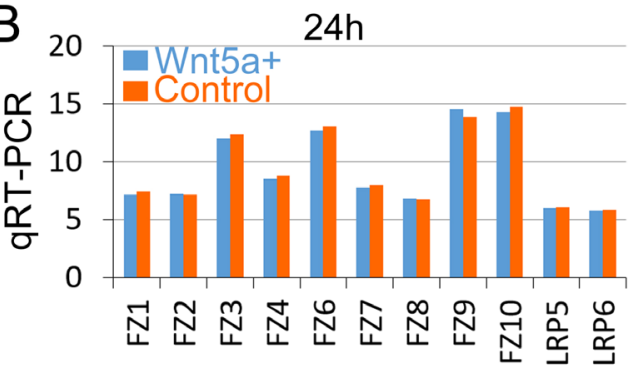

Bright
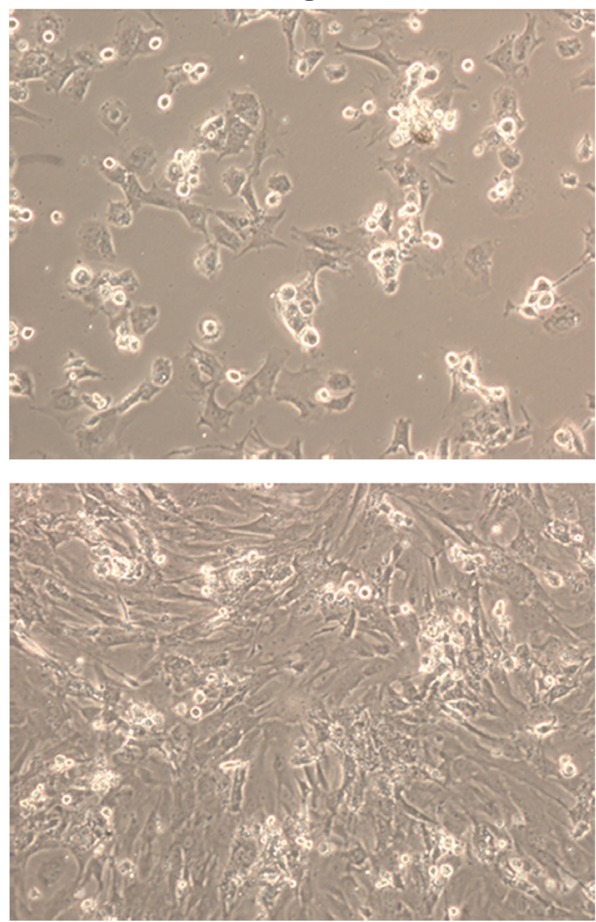

C

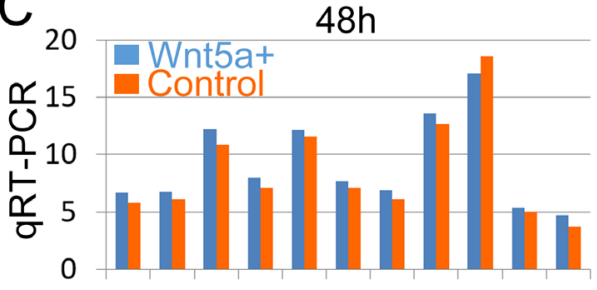

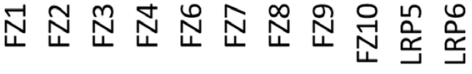
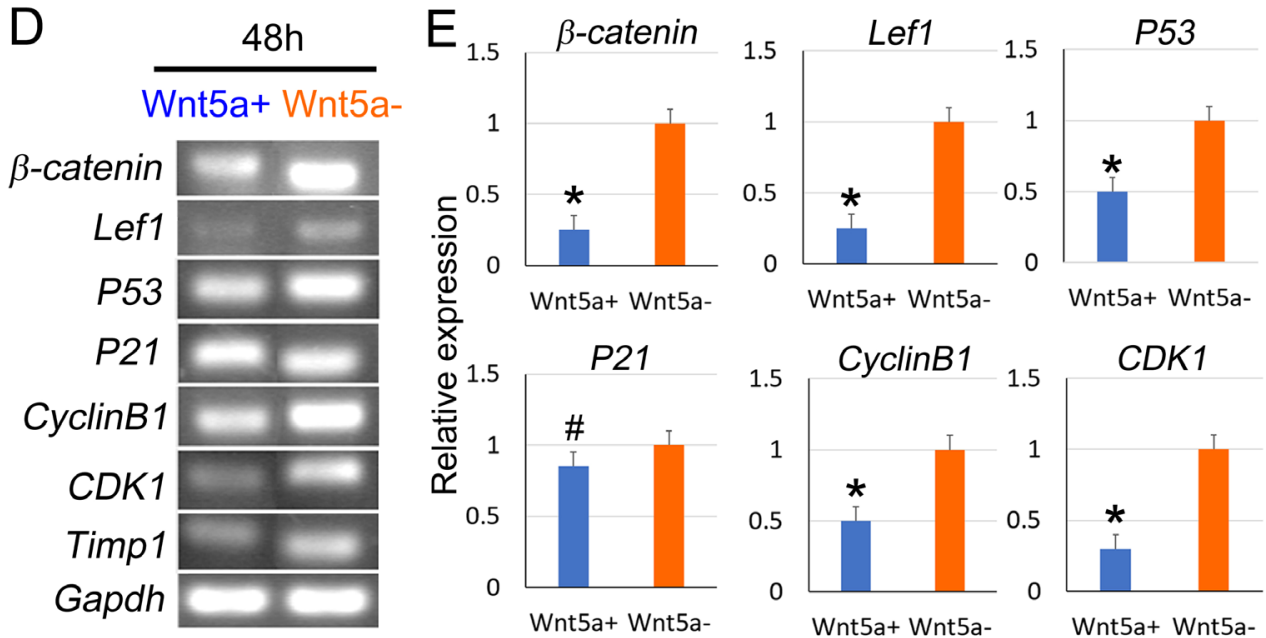

Figure 5: Overexpression of Wnt5a attenuates canonical Wnt signaling pathway in cultured DP cells. (A) Green fluorescence indicates successful infection of AdWnt5a and AdGFP control adenoviruses. (B) qRT-PCR shows that no significant change of Frizzled receptors expression in cultured DP cells 24h after Wnt5a treatment, compared to the control group. Blue bars represent Wnt5a overexpression groups, and deep yellow bars represent control groups. (C) qRT-PCR shows that no significant change of Frizzled receptors expression in cultured DP cells 48h after Wnt5a treatment, compared to the control group. (D) RT-PCR shows that expression of genes involved in Wnt pathway and cell cycling in cultured DP cells $48 \mathrm{~h}$ after Wnt5a treatment, compared to the control group. (E) Statistical analysis reveals differential expression of genes involved in Wnt pathway and cell cycling in cultured DP cells $48 \mathrm{~h}$ after Wnt5a treatment, compared to the control group. $\mathrm{P}<0.5$. $\mathrm{N}=3$. 
to regulate epithelial cell differentiation? Wnt5a is able to attenuates $\mathrm{Wnt} / \beta$-catenin signaling in cultured human DP cells [32], and suppresses Wnt/ $\beta$-catenin signaling during telogen-to-anagen transition. Our results also showed that overexpression of Wnt5a in DP cells results in decreased expression of canonical Wnt pathway genes such as $\beta$-catenin and Lef1. These studies suggest that $W n t 5 a$ is sufficient to inhibit canonical Wnt signaling pathway both in vivo and in vitro in mouse cells. Therefore, canonical Wnt signaling pathway genes are significantly increased $[12,24]$ in Gsdma3-mutant mice in which Wnt5a expression is decreased. Canonical Wnt signaling is important for regulating hair follicle differentiation. Thus, decreased Wnt5a expression results an aberrant hair shaft differentiation in the hair follicle.

In addition, it is obvious that the homeostasis of the DP is changed. Our PCR results show that P53 expression is significantly decreased in cultured DP cells after $W n t 5 a$ treatment. $P 53$ has various functions such as regulating DNA repair, cell aging, senescence and apoptosis. P53 binds to $P 21$ to establish a longer G1 phase during cell cycle [33]. However, in our study, we found that $P 21$ is not changed in DP cells after Wnt 5 a treatment, suggesting that P53 may not promote DP cell apoptosis in vitro. Indeed, we also didn't observe significant cell apoptosis in the DP cells in Gsdma3-mutant mice in vivo [25, 26]. On the other side, other cell cycle genes such as Cyclin
$B 1$ and $C D K 1$ that are involved in $\mathrm{G} 2 / \mathrm{M}$ phase are also decreased in DP cells after Wnt5a treatment, indicating Wnt5a may prevent DP cell proliferation. Previous study shows that Wnt5a suppresses hematopoietic stem cells by maintaining them in a quiescent G0 state [16]. P53 also plays important role in promoting embryonic stem cells differentiation [34]. Thus, decreased expression P53 in the DP cells after $W n t 5 a$ treatment may function to regulate maintaining DP cells in a more quiescent state. However, active cellular behaviors occur during hair follicle structure reestablishment during anagen phase, relying largely on epithelial-DP interaction. The relatively quiescent DP cells may also contribute to aberrant hair follicle differentiation in Gsdma3-mutant mice.

Taking together, our findings demonstrate that Gsdma3 promotes hair follicle differentiation by positively regulating $W n t 5 a$ expression in the epithelia of the hair follicle. Epithelia-derived Wnt5a further influences DP cells which govern the adjacent epithelial transient amplifying cells to differentiate into the hair shaft and IRS (Figure 6). How the molecular change of DP cells influences DP-epithelial interaction remains further investigation. Our study provides new clues not only for the relationship between canonical and non-canonical Wnt signaling pathways in regulating hair follicle growth, but also enhances the mechanism of epithelial-mesenchymal interactions in governing hair follicle differentiation.

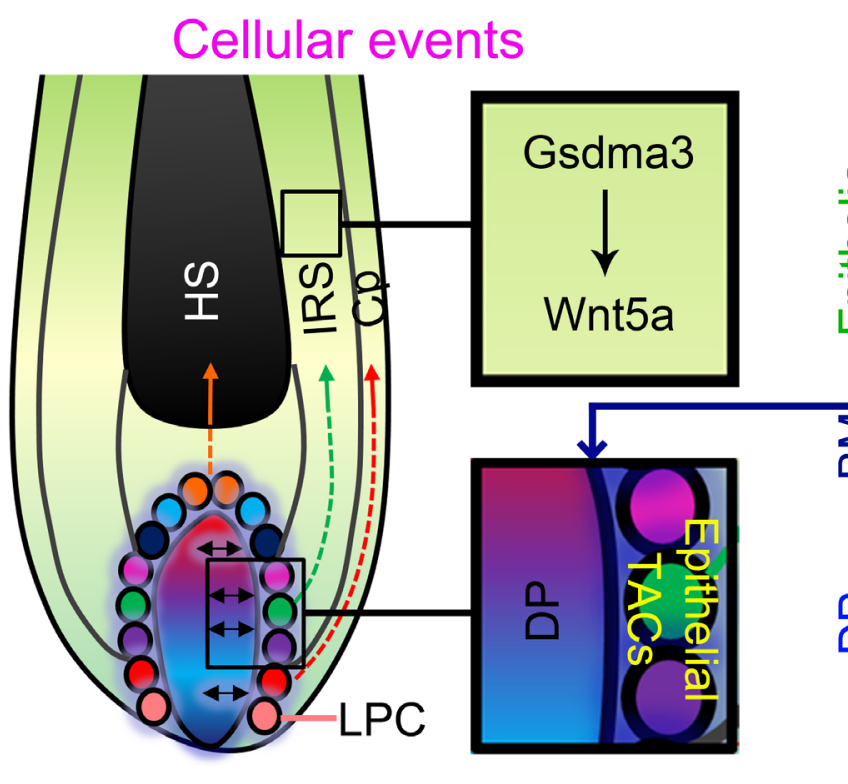

\section{Molecular events}
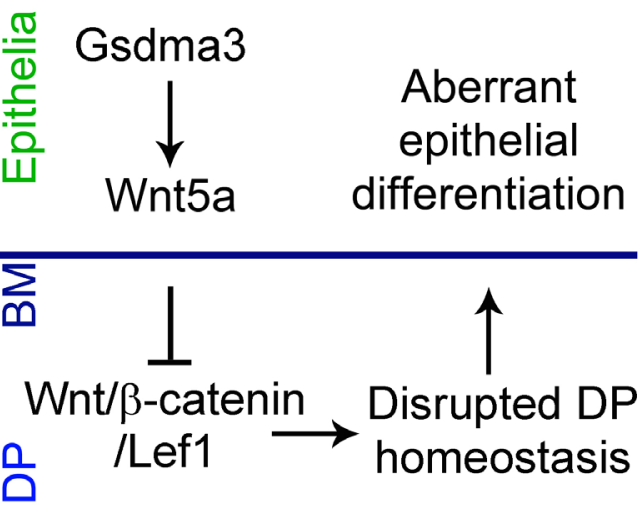

Epithelia-DP interaction

Figure 6: Epithelial-mesenchymal mechanism involved in hair follicle differentiation. Normal expression of $G s d m a 3$ results in a proper hair follicle differentiation. In Gsdma3-mutant mice, loss of function of Gsdma3 leads to decreased Wnt5a expression in epithelia of the hair follicle. Decreased Wnt5a in epithelia causes up-regulated canonical Wnt signaling pathway, leading to a disrupted homeostasis of the DP, which results in an aberrant epithelial differentiation. Whereas overexpression of Wnt5a results in decreased expression of canonical Wnt signaling pathway genes, which also leads to a disrupted homeostasis of the DP, in turn causing an aberrant epithelial differentiation. Colorful depicts represent the heterogeneity in TACs. BM, basement membrane; DP, dermal papilla; LPC, lower proximal cup; HS, hair shaft; IRS, inner root sheath; Cp, companion layer; TACs, transient amplifying cells. 


\section{MATERIALS AND METHODS}

Mice

Gsdma3-mutant mice were gifted from National Database of Mouse Genetic Resources, Nanjing, China. The mutant mice were generated from a point mutation (nt1112 T to C) on a C57BL/6 background by ENU (ethylnitrosourea). Both Gsdma3-mutant mice and C57BL/6 wild mice were housed at the Experimental Animal Center of the Third Military Medical University, China. All the experimental protocols were approved by the Research Committee of the Third Military Medical University.

\section{Histology and immunostaining}

Skin samples were harvested from the dorsal track of Gsdma3-mutant mice or C57BL/6 mice, and fixed in $4 \%$ paraformaldehyde in PBS for overnight. Sections were cut at $5 \mathrm{~mm}$, dewaxed by xylene, and rehydrated by ethanol in gradient concentrations. For hematoxylin-eosin (H\&E) staining, sections were stained with hematoxylin and eosin for $1 \mathrm{~min}$, respectively, and then mounted with resinene. For immunostaining, sections were subjected to antigen retrieval with $0.1 \mathrm{M}$ citric acid buffer at ph6.0. Then the samples were incubated with primary antibodies against aSMA (1:100, Boisynthesis Biotechnology Co., Ltd. Beijing, China), Wnt5a (1:1000, Abcam, Cambridge, USA) and Gsdma3 (1:100, GL Biochem, Shanghai, China) [24] at $4^{\circ} \mathrm{C}$ for overnight. After wash, the samples were incubated with Alexa Fluor 594 (1:500, Beyotime, Jiang $\mathrm{Su}$, China) conjugated secondary antibodies. Nucleus was labeled with 4', 6'-diamidino-2-phenylindole (DAPI, 1: 1,000, Sigma-Aldrich, St. Louis, MO, USA).

\section{Reverse transcription polymerase chain reaction (RT-PCR)}

Total RNA was extracted by using Trizol reagent (Life Technologies, Grand Island, USA). One ug of total RNA was reversely transcribed to cDNA by using a RT kit (Toyobo, Japan), at $30^{\circ} \mathrm{C}$ for $10 \mathrm{~min}, 42^{\circ} \mathrm{C}$ for $20 \mathrm{~min}$, $94^{\circ} \mathrm{C}$ for $10 \mathrm{~min}$, and $4^{\circ} \mathrm{C}$ for $5 \mathrm{~min}$ in order. The primers used in the PCR reactions were listed in Supplementary Table 1. The TM value for PCR was set at $63^{\circ} \mathrm{C}$.

\section{Plasmid and adenovirus}

Generation of Gsdma3 overexpression plasmid was as previously described [26]. In brief, CDS sequence of the Gsdma3 gene was cloned into a pEGFP-N1 plasmid, with a forward primer 5'-ACGCGTCGACATGCCT GTGTTTGAGGATGTCAC-3' and a reverse primer 5'-TCCCCGCGG AGATAGAGCACAATAAGTAAGT GCATTAGA-3'. Adenovirus-mediated overexpression of Wnt5a (AdWnt5a) and AdGFP (control) adenoviruses were gifted from Dr. Tong-Chuan He (University of Chicago, USA). Adenoviruses were propagated in HEK293 cells.

\section{Cell culture and infection}

For culture of hair follicle epithelial cells, vibrissa was dissected out by using a micro-needle from postnatal day 8 (P8) mice. After wash in PBS, the vibrissa was placed into a culture dish, and cultured in DMEM culture medium containing $10 \%$ fetal bovine serum (FBS).

JB6 Cl 30-7b (JB6 cells) mouse epithelial cell line was purchased (ATCC, Manassas, USA). JB6 cells were cultured in DMEM culture medium containing 10\% FBS. Four ug mouse recombinant Gsdma3 overexpression plasmid or pEGFP-N1 control plasmid was transfected to JB6 cells by using a lipofectamine 2000 kit (Life Technologies, Grand Island, USA). Cells were harvested for RNA extraction 48 hours after plasmid transfection.

For culture of DP cells, vibrissa was dissected out from P8 mice. After wash in Hanks solution, the vibrissa was treated with $0.25 \%$ dispase at $37^{\circ} \mathrm{C}$ for $40 \mathrm{~min}$. Then the DP was dissected out by using a micro needle, digested by $0.25 \%$ dispase at $37^{\circ} \mathrm{C}$ for 1.5 hours, and then centrifuged at $500 \mathrm{rpm}$ for $3 \mathrm{~min}$. The cells were resuspended and digested again by $0.25 \%$ dispase at $37^{\circ} \mathrm{C}$ for $30 \mathrm{~min}$. After being centrifuged at $2000 \mathrm{rpm}$ for $3 \mathrm{~min}$, the cells were seeded into a culture dish with DMEM culture medium containing $10 \%$ FBS.

\section{Alkaline phosphatase staining}

DP cells were passaged into a 24-well culture plate placed with small cover glasses. 24 hours after culture, the cells adhered to the cover glasses were washed in PBS for 3 times, and incubated with NBT/BCIP solution at room temperature for $10 \mathrm{~min}$.

\section{Statistical analysis}

All experiments were repeated at least three times. The statistical analysis was expressed at mean \pm SD. Student's t test was used to evaluate the statistical significance, which was set as $\mathrm{p}<0.05$.

\section{Abbreviations}

Ad: adenovirus; DP: dermal papilla; IRS: inner root sheath; ORS: outer root sheath; TACs: transient amplifying cells; Wnt: wingless-type MMTV integration site family.

\section{Author contributions}

Conception and design: LH, ML, PC, TY, YL, LY, WL, XL, YX

Development of methodology: LH, ML, YX, XL 
Acquisition of data: $\mathrm{LH}, \mathrm{ML}, \mathrm{XL}, \mathrm{CH}$

Analysis and interpretation of data: LH, ML, XL

Writing, review, and/or revision of the manuscript: ML.

\section{CONFLICTS OF INTEREST}

The authors state no conflicts of interest.

\section{FINACIAL SUPPORT}

This study is supported by Innovation and Attracting Talents Program for College and University (' 111 ' Project) (B06023), and National Nature Science Foundation of China (11532004, 31270990). Mingxing Lei is supported by projects funded by China Postdoctoral Science Foundation (2016M590866), Fundamental Research Funds for the Central Universities (106112015CDJRC231206), Special Funding for Postdoctoral Research Projects in Chongqing (Xm2015093), and a fellowship from the China Scholarship Council (2011605042).

\section{REFERENCES}

1. Ribatti D, Santoiemma M. Epithelial-mesenchymal interactions: a fundamental Developmental Biology mechanism. Int J Dev Biol. 2014; 58: 303-6. https://doi. org/10.1387/ijdb.140143dr.

2. Lei M, Schumacher LJ, Lai YC, Juan WT, Yeh CY, Wu P, Jiang TX, Baker RE, Widelitz RB, Yang L, Chuong CM. Self-organization process in newborn skin organoid formation inspires strategy to restore hair regeneration of adult cells. Proc Natl Acad Sci U S A. 2017; 114: E7101-E10. https://doi.org/10.1073/pnas.1700475114.

3. Sennett R, Rendl M. Mesenchymal-epithelial interactions during hair follicle morphogenesis and cycling. Semin Cell Dev Biol. 2012; 23: 917-27. https://doi.org/10.1016/j. semcdb.2012.08.011.

4. Lei M, Chuong CM. STEM CELLS. Aging, alopecia, and stem cells. Science. 2016; 351: 559-60. https://doi. org/10.1126/science.aaf1635.

5. Chueh SC, Lin SJ, Chen CC, Lei MX, Wang LM, Widelitz R, Hughes MW, Jiang TX, Chuong CM. Therapeutic strategy for hair regeneration: hair cycle activation, niche environment modulation, wound-induced follicle neogenesis, and stem cell engineering. Expert Opin Biol Ther. 2013; 13: 377-91. https://doi.org/10.1517/14712598 .2013 .739601 .

6. Wang N, Yang T, Li J, Lei M, Shi J, Qiu W, Lian X. The expression and role of c-Myc in mouse hair follicle morphogenesis and cycling. Acta Histochem. 2012; 114: 199-206. https://doi.org/10.1016/j.acthis.2011.04.009.

7. Lei MX, Chuong CM, Widelitz RB. Tuning Wnt signals for more or fewer hairs. J Invest Dermatol. 2013; 133: 7-9. https://doi.org/10.1038/jid.2012.446.
8. Ouspenskaia T, Matos I, Mertz AF, Fiore VF, Fuchs E. WNT-SHH antagonism specifies and expands stem cells prior to niche formation. Cell. 2016; 164: 156-69. https:// doi.org/10.1016/j.cell.2015.11.058.

9. Qiu W, Tang H, Guo H, Lei M, Yan H, Lian X, Wu J. 12-O-tetradecanoylphorbol-13-acetate activates hair follicle melanocytes for hair pigmentation via Wnt/beta-catenin signaling. Cell Tissue Res. 2016; 366: 329-40. https://doi. org/10.1007/s00441-016-2450-6.

10. Lei MX, Lai XD, Bai XF, Qiu WM, Yang T, Liao XL, Chuong CM, Yang L, Lian XH, Zhong JL. Prolonged overexpression of Wnt10b induces epidermal keratinocyte transformation through activating EGF pathway. Histochem Cell Biol. 2015; 144: 209-21. https://doi.org/10.1007/ s00418-015-1330-6.

11. Lei MX, Guo HY, Qiu WM, Lai XD, Yang T, Widelitz RB, Chuong CM, Lian XH, Yang L. Modulating hair follicle size with Wnt10b/DKK1 during hair regeneration. Exp Dermatol. 2014; 23: 407-13. https://doi.org/10.1111/ exd.12416.

12. Lei M, Yang T, Lai X, Bai X, Qiu W, Lian X, Yang L. Upregulation of interfollicular epidermal and hair infundibulum beta-catenin expression in Gsdma3 mutant mice. Acta Histochem. 2013; 115: 63-9. https://doi. org/10.1016/j.acthis.2012.04.002.

13. Xing Y, Ma X, Guo H, Deng F, Yang J, Li Y. Wnt5a suppresses beta-catenin signaling during hair follicle regeneration. Int J Med Sci. 2016; 13: 603-10. https://doi. org/10.7150/ijms.15571.

14. Ho HY, Susman MW, Bikoff JB, Ryu YK, Jonas AM, Hu L, Kuruvilla R, Greenberg ME. Wnt5a-Ror-Dishevelled signaling constitutes a core developmental pathway that controls tissue morphogenesis. Proc Natl Acad Sci U S A. 2012; 109: 4044-51. https://doi.org/10.1073/ pnas. 1200421109 .

15. Kessenbrock K, Smith P, Steenbeek SC, Pervolarakis N, Kumar R, Minami Y, Goga A, Hinck L, Werb Z. Diverse regulation of mammary epithelial growth and branching morphogenesis through noncanonical Wnt signaling. Proc Natl Acad Sci U S A. 2017; 114: 3121-6. https://doi. org/10.1073/pnas.1701464114.

16. Nemeth MJ, Topol L, Anderson SM, Yang Y, Bodine DM. Wnt5a inhibits canonical Wnt signaling in hematopoietic stem cells and enhances repopulation. Proc Natl Acad Sci U S A. 2007; 104: 15436-41. https://doi.org/10.1073/ pnas.0704747104.

17. Stefater JA 3rd, Lewkowich I, Rao S, Mariggi G, Carpenter AC, Burr AR, Fan J, Ajima R, Molkentin JD, Williams BO, Wills-Karp M, Pollard JW, Yamaguchi T, et al. Regulation of angiogenesis by a non-canonical Wnt-Flt1 pathway in myeloid cells. Nature. 2011; 474: 511-5. https://doi. org/10.1038/nature10085.

18. Reddy S, Andl T, Bagasra A, Lu MM, Epstein DJ, Morrisey EE, Millar SE. Characterization of Wnt gene expression in developing and postnatal hair follicles and identification 
of Wnt5a as a target of Sonic hedgehog in hair follicle morphogenesis. Mech Dev. 2001; 107: 69-82.

19. Huang S, Zhu X, Liu Y, Tao Y, Feng G, He L, Guo X, Ma G. Wls is expressed in the epidermis and regulates embryonic hair follicle induction in mice. PLoS One. 2012; 7: e45904. https://doi.org/10.1371/journal.pone.0045904.

20. Xing YZ, Wang RM, Yang K, Guo HY, Deng F, Li YH, Ye JX, He L, Lian XH, Yang T. Adenovirus-mediated Wnt5a expression inhibits the telogen-to-anagen transition of hair follicles in mice. Int J Med Sci. 2013; 10: 908-14. https:// doi.org/10.7150/ijms.6137.

21. Zhu X, Wu Y, Huang S, Chen Y, Tao Y, Wang Y, He S, Shen $\mathrm{S}$, Wu J, Guo X, Li B, He L, Ma G. Overexpression of Wnt5a in mouse epidermis causes no psoriasis phenotype but an impairment of hair follicle anagen development. Exp Dermatol. 2014; 23: 926-8. https://doi.org/10.1111/ exd.12539.

22. Xing Y, Xu W, Yang K, Lian X, Yang T. Immunolocalization of Wnt5a during the hair cycle and its role in hair shaft growth in mice. Acta Histochem. 2011; 113: 608-12. https:// doi.org/10.1016/j.acthis.2010.06.006.

23. Hu B, Lefort K, Qiu W, Nguyen BC, Rajaram RD, Castillo E, He F, Chen Y, Angel P, Brisken C, Dotto GP. Control of hair follicle cell fate by underlying mesenchyme through a CSL-Wnt5a-FoxN1 regulatory axis. Genes Dev. 2010; 24: 1519-32. https://doi.org/10.1101/gad.1886910.

24. Bai XF, Lei MX, Shi JZ, Yu Y, Qiu WM, Lai XD, Liu YX, Yang T, Yang L, Widelitz RB, Chuong CM, Lian XH. Roles of GasderminA3 in Catagen-Telogen Transition during Hair Cycling. J Invest Dermatol. 2015; 135: 2162-72. https://doi. org/10.1038/jid.2015.147.

25. Lei M, Gao X, Yang L, Yang T, Lian X. Gsdma3 gene is needed for the induction of apoptosis-driven catagen during mouse hair follicle cycle. Histochem Cell Biol. 2011; 136: 335-43. https://doi.org/10.1007/s00418-011-0845-8.

26. Lei M, Bai X, Yang T, Lai X, Qiu W, Yang L, Lian X. Gsdma3 is a new factor needed for TNF-alpha-mediated apoptosis signal pathway in mouse skin keratinocytes.
Histochem Cell Biol. 2012; 138: 385-96. https://doi. org/10.1007/s00418-012-0960-1.

27. Li J, Zhou Y, Yang T, Wang N, Lian X, Yang L. Gsdma3 is required for hair follicle differentiation in mice. Biochem Biophys Res Commun. 2010; 403: 18-23. https://doi. org/10.1016/j.bbrc.2010.10.094.

28. Yang H, Adam RC, Ge Y, Hua ZL, Fuchs E. Epithelialmesenchymal micro-niches govern stem cell lineage choices. Cell. 2017; 169: 483-96 e13. https://doi. org/10.1016/j.cell.2017.03.038.

29. Tamura M, Tanaka S, Fujii T, Aoki A, Komiyama H, Ezawa K, Sumiyama K, Sagai T, Shiroishi T. Members of a novel gene family, Gsdm, are expressed exclusively in the epithelium of the skin and gastrointestinal tract in a highly tissue-specific manner. Genomics. 2007; 89: 618-29. https:// doi.org/10.1016/j.ygeno.2007.01.003.

30. Kastan MB, Zhan Q, el-Deiry WS, Carrier F, Jacks T, Walsh WV, Plunkett BS, Vogelstein B, Fornace AJ Jr. A mammalian cell cycle checkpoint pathway utilizing p53 and GADD45 is defective in ataxia-telangiectasia. Cell. 1992; 71: 587-97.

31. Gartel AL, Radhakrishnan SK. Lost in transcription: $\mathrm{p} 21$ repression, mechanisms, and consequences. Cancer Res. 2005; 65: 3980-5. https://doi.org/10.1158/0008-5472. CAN-04-3995.

32. Kwack MH, Kim MK, Kim JC, Sung YK. Wnt5a attenuates Wnt/beta-catenin signalling in human dermal papilla cells. Exp Dermatol. 2013; 22: 229-31. https://doi.org/10.1111/ exd.12101.

33. Agarwal ML, Agarwal A, Taylor WR, Stark GR. p53 controls both the G2/M and the G1 cell cycle checkpoints and mediates reversible growth arrest in human fibroblasts. Proc Natl Acad Sci U S A. 1995; 92: 8493-7.

34. Maimets T, Neganova I, Armstrong L, Lako M. Activation of p53 by nutlin leads to rapid differentiation of human embryonic stem cells. Oncogene. 2008; 27: 5277-87. https://doi.org/10.1038/onc.2008.166. 